\title{
Lack of MEF2A mutations in coronary artery disease
}

Li Weng, ${ }^{1}$ Nihan Kavaslar, ${ }^{2}$ Anna Ustaszewska, ${ }^{1}$ Heather Doelle, ${ }^{2}$ Wendy Schackwitz, ${ }^{1}$ Sybil Hébert, ${ }^{2}$ Jonathan C. Cohen, ${ }^{3}$ Ruth McPherson, ${ }^{2}$ and Len A. Pennacchio ${ }^{1,4}$

\author{
1US Department of Energy, Joint Genome Institute, Walnut Creek, California, USA. 2Division of Cardiology and \\ the Lipoprotein and Atherosclerosis Research Group, University of Ottawa Heart Institute, Ottawa, Ontario, Canada. \\ ${ }^{3}$ Center for Human Nutrition and McDermott Center for Human Growth and Development, University of Texas Southwestern Medical Center, \\ Dallas, Texas, USA. ${ }^{4}$ Genomics Division, Lawrence Berkeley National Laboratory, Berkeley, California, USA.
}

\begin{abstract}
Mutations in $M E F 2 A$ have been implicated in an autosomal dominant form of coronary artery disease (adCAD1). In this study we sought to determine whether severe mutations in MEF2A might also explain sporadic cases of coronary artery disease (CAD). To do this, we resequenced the coding sequence and splice sites of $M E F 2 A$ in approximately 300 patients with premature CAD and failed to find causative mutations in the CAD cohort. However, we did identify the 21-bP MEF2A coding sequence deletion originally implicated in adCAD1 in 1 of 300 elderly control subjects without CAD. Further screening of approximately 1,500 additional individuals without CAD revealed 2 more subjects with the MEF2A 21-bp deletion. Genotyping of 19 family members of the 3 probands with the 21-bp deletion in MEF2A revealed that the mutation did not cosegregate with early CAD. These studies support that MEF2A mutations are not a common cause of CAD in white people and argue strongly against a role for the MEF2A 21-bp deletion in autosomal dominant CAD.
\end{abstract}

\section{Introduction}

Coronary artery disease (CAD) is one of the leading causes of mortality in Western societies (1). Like other common chronic diseases, CAD has a complex etiology that is postulated to involve both genetic and environmental factors. Several risk factors for CAD have been established, including family history, hypertension, dyslipidemia, obesity, smoking, diabetes, and diet (2-5). In addition, genetic association studies and genome-wide linkage scans have uncovered several susceptibility loci and candidate genes that might contribute to the pathogenesis of CAD (6-13), although many of these studies remain controversial $(14,15)$.

Recently, a mutation in the human MEF2A gene, a member of the myocyte enhancer family of transcription factors, has been implicated in an autosomal dominant form of CAD (adCAD1) (16). Genetic linkage analysis of a single large white family with an autosomal dominant pattern of premature CAD indicated positive linkage (log odds ratio [LOD], 4.19) to a single locus on chromosome 15 q26 that included approximately 90 annotated genes. Resequencing of $M E F 2 A$, a prime candidate gene in the linked locus (17-19), revealed a 21-bp coding sequence deletion in all affected family members. The predicted 7 -amino acid deletion was found to occur in a region conserved among MEF2A proteins in humans, mice, pigs, and spider monkeys, and in vitro assays of $M E F 2 A$ function suggested that the deletion disrupts nuclear localization of the mutant protein and reduces MEF2Ainduced transcriptional activation. No $M E F 2 A$ mutations were identified in 3 additional large families with prevalent CAD or 50 additional sporadic patients with this common disease. However, since the completion of the original study, the authors

Nonstandard abbreviations used: adCAD1, autosomal dominant form of coronary artery disease; $\mathrm{CAD}$, coronary artery disease.

Conflict of interest: The authors have declared that no conflict of interest exists.

Citation for this article: J. Clin. Invest. 115:1016-1020 (2005).

doi:10.1172/JCI200524186. have reported MEF2A missense mutations (N263S, P279L, and G283D) in 4 out of 207 sporadic cases of CAD and estimate that approximately $2 \%$ of CAD is due to mutations in this gene (20).

\section{Results}

To further explore the role of MEF2A mutations in the pathogenesis of nonfamilial cases of CAD, we resequenced the exons and flanking intron sequences of MEF2A in 300 white individuals with documented CAD with onset before the age of 55 years (men) or 65 years (women). Missense changes observed in the CAD group were examined in 300 elderly control subjects (men, older than 60 years; women, older than 70 years) who did not have signs or symptoms of CAD, and only 1 isolated change (S360L) was found to be unique to the CAD cohort. Computational analysis using PolyPhen (21) predicted that this change is benign, and additional examination of deep vertebrate protein alignments further revealed that this is not a constrained position in MEF2A or other paralogous protein family members $(22,23)$. The absence of severe mutations in MEF2A in our 300 CAD patients suggests that mutations in $M E F 2 A$ are not a major cause of sporadic forms of CAD in white people, in contrast to the recent finding of M.R.K. Bhagavatula et al. (20).

Resequencing did reveal a complex coding sequence length polymorphism in the last coding exon of MEF2A that results from tri-nucleotide length variants within a region of polyglutamine and -proline repeats (Figure 1). Analyses of the repeat region in 287 CAD samples and 296 controls revealed 9 length-variant alleles overlapping the polyglutamine repeat in our sample set. All 9 alleles occurred at similar frequencies in CAD patients and control subjects (Table 1). We also identified 2 length-variant alleles in an adjacent polyproline repeat ( 5 versus 4 prolines); however, more than $98 \%$ of individuals in both CAD and control classes contained 5 prolines, yielding no statistically significant difference between the 2 groups. These data support that there is no association between these length 


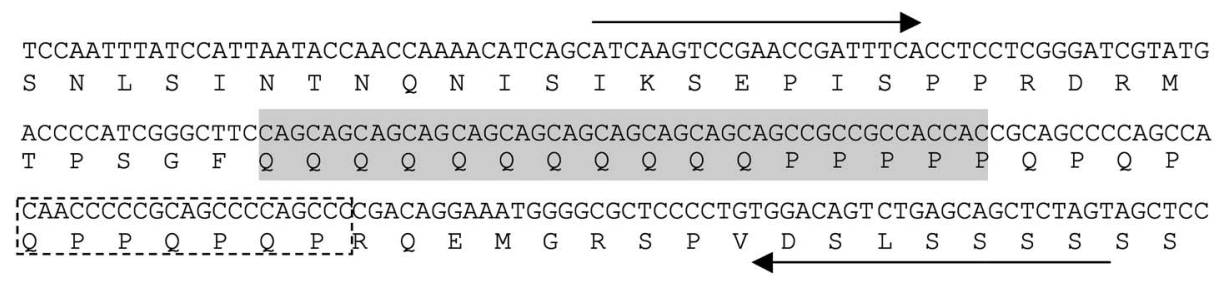

Figure 1

Nucleotide and amino acid sequences of the repeat region in MEF2A. The shaded box marks the polyglutamine and -proline tandem repeats; the 21-bp deletion is boxed with dashed lines; arrows indicate the primers used for fragment-size analysis.

polymorphisms in MEF2A and CAD susceptibility in this sample set of white individuals.

Whereas none of the CAD patients in this study had obvious $M E F 2 A$ mutations, the 21-bp deletion originally implicated in adCAD1 (16) was identified in one of the elderly control subjects (control 1) (Figure 2), a 71-year-old with no symptoms of CAD. The deletion did not segregate with CAD in the subject's family (Table 2 and Figure 3). Notably, in this kindred, 3 elderly subjects with the 21-bp deletion did not have evidence of premature CAD, whereas the 2 subjects with premature CAD did not carry the deletion. This finding suggested that the 21-bp deletion may not be a disease-causing mutation.

To further explore this possibility, we screened for the 21-bp deletion in MEF2A in an additional 1,521 unrelated white individuals who did not have a history of or symptoms suggestive of possible CAD. Two additional subjects (controls 2 and 3 ) were found to carry the deletion (Figure 2), and in both cases the deletion did not segregate with CAD in their extended families. Overall, among the family members of controls 1, 2, and 3, we identified 4 subjects bearing the 21-bp deletion who were older than 60 years of age and had other major CAD risk factors, including dyslipidemia, hypertension, diabetes, and a history of smoking. However, none of these subjects had evidence of CAD. Since the 21-bp deletion has been reported to have a dominant negative effect on the transcriptional activity of wild-type MEF2A (16) and since mice with inactivation of Mef2a display marked right ventricular hypertrophy (19), we also performed 2-dimensional echocardiography on 5 control subjects bearing the 21-bp deletion. These included a 71-yearold female (control 1) (II-6 in kindred no. 1, Table 2), a 45-yearold obese male with type 2 diabetes (control 2), a 45-year-old normal-weight male (control 3), and subjects III-5 and III-6 from kindred no. 1. All individuals had normal right and left ventricular size and function.

\section{Discussion}

Taken together, our data indicate that missense mutations and length polymorphisms in $M E F 2 A$ do not contribute appreciably to CAD in the general population. Furthermore, the 21-bp deletion in $M E F 2 A$, which has a frequency of approximately $0.15 \%$ in the population, does not appear to be associated with premature CAD. Although we cannot completely rule out the possibility that the 21-bp deletion in MEF2A might affect susceptibility to CAD with variable penetrance, the finding that this allele did not segregate with the disease in 3 independent families strongly suggests that it does not cause an autosomal dominant form of CAD.

\section{Methods}

Subjects and DNA isolation. CAD patients $(n=300)$ were recruited from the patient population of the University of Ottawa Heart Institute. CAD was defined as documented myocardial infarction and/or angiographic evidence of at least one coronary artery with more than $50 \%$ stenosis. The majority of subjects had a history of severe CAD and had undergone coronary artery bypass grafting. Criteria for inclusion in the study included an age of onset of CAD less than 55 years for men and less than 65 years for women, no history of diabetes, and absence of monogenic lipid disorders and severe dyslipidemia (total cholesterol prior to treatment, less than $7.0 \mathrm{mmol} / \mathrm{l}$ or less than $270 \mathrm{mg} / \mathrm{dl}$ ). Elderly control subjects $(n=300)$, including men older than 60 years and women older than 70 years, with no history or symptoms of CAD were recruited from the Ottawa area. A second cohort of 1,521 control subjects, mean age 46 years, including both obese and normal-weight subjects, without a history of premature CAD was recruited separately from the Ottawa region. Families of 3 unrelated subjects from the above cohorts who were found to bear the MEF2A 21-bp deletion were also studied. These included 9 members of kindred no. 1, 9 members of kindred no. 2, and 4 members of kindred no. 3. This study was approved by the Institutional Review Board of the University of Ottawa Heart Institute, and informed written consent was obtained from all participants. Genomic DNA was extracted from white blood cells by standard methods (24) and from saliva samples collected in Oragene kits by the manufacturer's instructions (DNA Genotek Inc.).

Sequencing and data analysis. Primers were designed to give a maximum product size of $500 \mathrm{bp}$ and a minimum of $40 \mathrm{bp}$ flanking the splice sites, using the Exon Locator and eXtractor for Resequencing program version 2.0 (25). An M13F tag (GTTTTCCCAGTCACGACGTTGTA) and an M13R tag (AGGAAACAGCTATGACCAT) were added to forward and reverse primers, respectively. Ten nanograms of DNA from each sample was amplified in a 10- $\mu$ l PCR reaction using AmpliTaq Gold (Applied Biosystems) and cleaned using CleanSEQ (Agencourt) (26). Sequencing reactions were performed using the M13 primers along with a BigDye

\section{Table 1}

Analysis of the allele distribution of the polyglutamine repeat length polymorphism in the CAD and control populations

$\begin{array}{lcccccc}\text { Allele }^{A} & \mathbf{1 1} & \mathbf{1 0} & \mathbf{9} & \mathbf{0 t h e r} & \text { Total } & \chi^{2} \text { (P value) } \\ \mathrm{N}_{\text {CAD }} & 292 & 76 & 197 & 9 & 574 & \\ \mathrm{~N}_{\text {CON }} & 288 & 80 & 217 & 7 & 592 & 1.056(0.787) \\ \text { Total } & 580 & 156 & 414 & 16 & 1166 & \end{array}$

ANumber of polyglutamine repeats. $\mathrm{N}_{\mathrm{CAD}}$, number of alleles in individuals with $\mathrm{CAD}$; $\mathrm{N}_{\mathrm{CON}}$, number of alleles in control subjects. "Other" includes rare alleles with $5,6,7,8,12$, or 13 glutamines. 

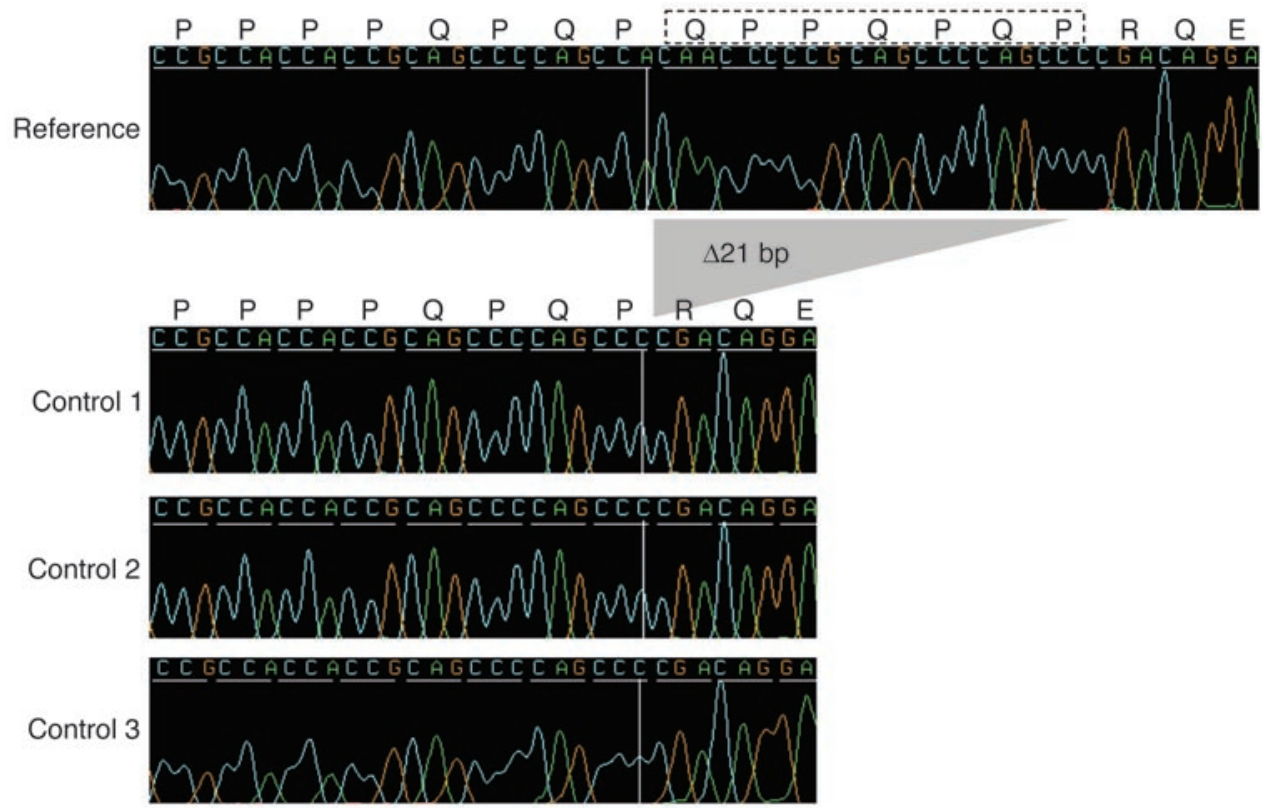

Figure 2

Sequence of the 21-bp deletion region of MEF2A. The PCR products from common and deletion alleles were separated by $3 \%$ agarose gel, purified, and cloned for sequencing analysis. The 21-bp deletion was identified in 3 independent control subjects. The 7 deleted amino acids are depicted with a dashed-line box. Haplotype analysis of all 3 subjects with the 21 -bp deletion as well as approximately 370 control subjects indicates that this variant resides on a common haplotype, consistent with the deletion arising through a single ancestral founding event (data not shown).

Terminator v3.1 Cycle Sequencing Kit (Applied Biosystems) (27) and cleaned again with CleanSEQ before being run on a 3730xl DNA Analyzer (Applied Biosystems). Base calling, quality assessment, and assem- bly were carried out using the Phred, Phrap, PolyPhred, Consed software suite $(28,29)$. All sequence variants identified were verified by manual inspection of the chromatograms by 2 individuals to ensure accuracy.

\section{Table 2}

Clinical characteristics of family members in kindred no. 1

\begin{tabular}{|c|c|c|c|c|c|c|c|c|c|c|c|c|c|}
\hline ID no. & $\begin{array}{l}\text { Current age or } \\
\text { age at death }\end{array}$ & Sex & $\begin{array}{c}\text { Premature } \\
\text { CADA }\end{array}$ & $\begin{array}{c}\text { 21-bp } \\
\text { deletion }\end{array}$ & $\begin{array}{c}\mathrm{TC} \\
(\mathrm{mg} / \mathrm{dl})\end{array}$ & $\begin{array}{c}\mathrm{TG} \\
(\mathrm{mg} / \mathrm{dl})\end{array}$ & $\begin{array}{l}\text { LDL-C } \\
\text { (mg/dl) }\end{array}$ & $\begin{array}{l}\text { HDL-C } \\
(\mathrm{mg} / \mathrm{dl})\end{array}$ & $\begin{array}{c}\text { TC/HDL-C } \\
\text { ratio }\end{array}$ & HTN & Smoker & Diabetes & Other \\
\hline $\mid-1$ & $67 y^{\mathrm{B}}$ (cancer) & $M$ & No & & - & - & - & - & - & - & - & - & - \\
\hline $1-2$ & $78 y^{B}$ (SD) & $F$ & No & & - & - & - & - & - & - & - & - & - \\
\hline$\|-1$ & $59 \mathrm{y}^{\mathrm{B}}$ (cancer) & M & No & & - & - & - & - & - & - & - & - & - \\
\hline II-2 & $58 \mathrm{y}^{\mathrm{B}}$ (cancer) & $\mathrm{F}$ & No & & - & - & - & - & - & - & - & - & - \\
\hline II-3 & & M & NK & & - & - & - & - & - & - & - & - & - \\
\hline$\|-4$ & $3 \mathrm{y}^{\mathrm{B}}$ (cancer) & M & NK & & - & - & - & - & - & - & - & - & - \\
\hline $11-5$ & $25 \mathrm{y}^{\mathrm{B}}$ (cancer) & M & NK & & - & - & - & - & - & - & - & - & - \\
\hline |I-6 & $71 \mathrm{y}$ & $\mathrm{F}$ & No & $+1-$ & 201 & 249 & 120 & 32 & 6.3 & Yes & No & T2DM & TIA at $69 \mathrm{y}$ \\
\hline $11-7$ & $46 y^{\mathrm{B}}$ (stroke) & M & NK & & - & - & - & - & - & - & - & - & - \\
\hline $11-8$ & $69 y$ & M & No & $-1-$ & 262 & 210 & 188 & 33 & 7.9 & Yes & No & T2DM & - \\
\hline II-9 & $67 y$ & M & No & & - & - & - & - & - & - & - & T2DM & - \\
\hline II-10 & $65 y$ & $\mathrm{M}$ & No & & - & - & - & - & - & - & - & - & - \\
\hline |I-11 & $64 \mathrm{y}$ & $\mathrm{F}$ & No & $+/-$ & 261 & 243 & 173 & 40 & 6.5 & No & No & IFG & - \\
\hline II-12 & $61 y$ & $\mathrm{M}$ & No & $+/-$ & 354 & 269 & 266 & 34 & 10.4 & Yes & No & T2DM & NSE \\
\hline III-1 & $57 \mathrm{y}$ & $\mathrm{F}$ & Yes & $-1-$ & 170 & 289 & 87 & 26 & 6.5 & No & Yes & - & - \\
\hline III-2 & $50 y$ & $M$ & No & $-1-$ & 229 & 166 & 167 & 29 & 7.9 & Yes & Ex & - & - \\
\hline III-3 & $48 y$ & $\mathrm{M}$ & Yes & $-1-$ & 217 & 116 & 155 & 39 & 5.6 & Yes & Ex & - & - \\
\hline |II-4 & $44 \mathrm{y}$ & $M$ & NK & & - & - & - & - & - & - & - & - & - \\
\hline III-5 & $42 \mathrm{y}$ & $\mathrm{F}$ & NK & $+1-$ & 252 & 87 & 194 & 41 & 6.1 & No & Yes & - & NSE \\
\hline III-6 & $37 y$ & $\mathrm{M}$ & NK & $+1-$ & 194 & 392 & 85 & 31 & 6.3 & No & No & - & NSE \\
\hline III-7 & $30 y$ & $\mathrm{~F}$ & NK & & - & - & - & - & - & - & - & - & - \\
\hline
\end{tabular}

APremature CAD defined as onset before age 50 years (males) or before age 55 years (females). ${ }^{B}$ Deceased. SD, sudden death; NK, early CAD status not known (male, younger than 50 years; female, younger than 55 years); TC, total cholesterol; TG, triglycerides; LDL-C, LDL cholesterol; HDL-C, HDL cholesterol; HTN, hypertension; T2DM, type 2 diabetes; IFG, impaired fasting glucose; TIA, transient ischemic attack; NSE, negative stress electrocardiogram. 


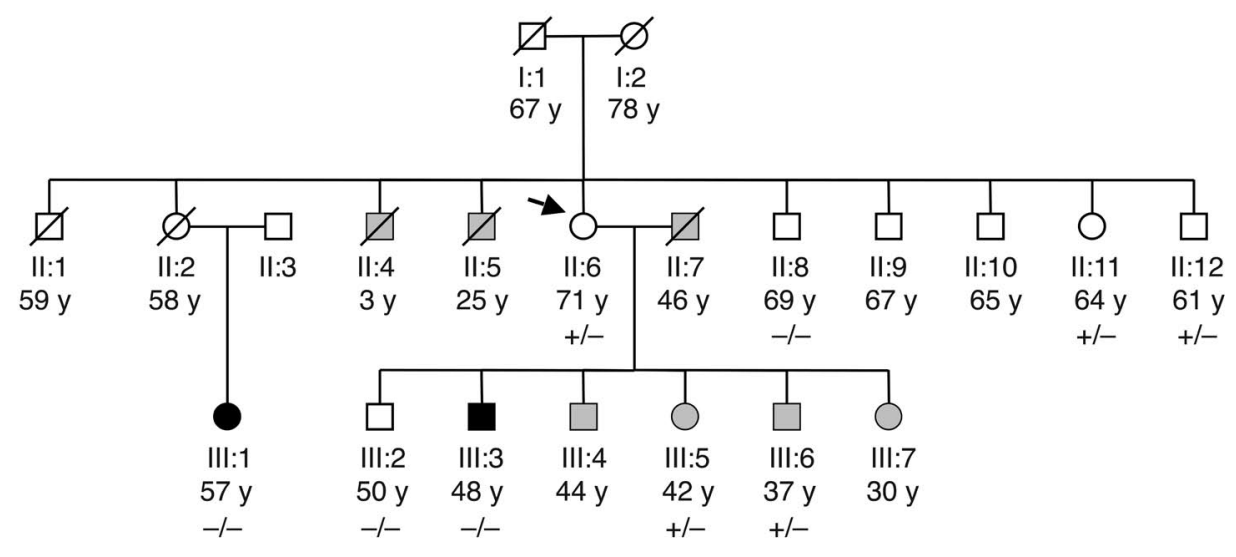

\section{Figure 3}

MEF2A 21-bp deletion does not cosegregate with CAD in kindred no. 1 (Table 2). Individuals with premature CAD are indicated by filled squares (males) or circles (females). Unaffected individuals are indicated by open squares or circles. Normal males under the age of 50 years and normal females under the age of 55 years are shown in light gray, which indicates uncertain phenotype. Deceased individuals are indicated by a slash. The proband is indicated by an arrow. Genetic status: +/- indicates the presence of the 21-bp deletion of MEF2A (heterozygous); $-/-$ indicates the absence of the deletion. Note that 3 elderly subjects with the 21-bp deletion do not have premature CAD, whereas the 2 subjects with premature CAD do not carry the deletion.

Fragment-size analysis. Fluorescently labeled PCR primers were designed to amplify approximately $180 \mathrm{bp}$ of a poly-glutamine and -proline repeat region within the $M E F 2 A$ gene (forward primer: FAM-ATCAAGTCCGAACCGATTTCA; reverse primer: ACTAGAGCTGCTCAGACTGTCCA). Following PCR, a 1:100 dilution of the PCR product was performed with water. Aliquots of $1.5 \mu \mathrm{l}$ of the diluted PCR product were then mixed with $0.2 \mu \mathrm{l}$ of the 400HD ROX Size Standard (Applied Biosystems) and $8.3 \mu \mathrm{l}$ of Hi-Di formamide (Applied Biosystems) before loading onto an ABI PRISM 3700 DNA Analyzer (Applied Biosystems). Gel images were analyzed using GeneScan Analysis Software version 3.7 (Applied Biosystems), and the fragment sizes were called using Genotyper Software version 3.7 (Applied Biosystems). To independently evaluate the fragment size, we genotyped the same repeat region by direct sequencing.

\section{Acknowledgments}

We would like to thank Jan-Fang Cheng and the Joint Genome Institute's production sequencing group for technical assistance, Edward Rubin and James Bristow for critical feedback, Robert Dent for assistance in recruitment of the control subjects, and the many individuals who participated in this study. This work was supported in part by Department of Energy Contract DEAC0378SF00098 (to L.A. Pennacchio) administered through the University of California; the Donald W. Reynolds Cardiovascular Clinical Research Center at Dallas; and Canadian Institutes of Health Research grant MOP-44360 and Heart and Stroke Foundation of Ontario grant NA-5413 (to R. McPherson).

Received for publication December 14, 2004, and accepted in revised form January 25, 2005.

Address correspondence to: Len A. Pennacchio, Genomics Division, One Cyclotron Road, MS 84-171, Lawrence Berkeley National Laboratory, Berkeley, California 94720, USA. Phone: (510) 486-7498; Fax: (510) 486-4229; E-mail: LAPennacchio@lbl. gov. Or to: Ruth McPherson, University of Ottawa Heart Institute, 40 Ruskin Street, Ottawa, Ontario K1Y 4W7, Canada. Phone: (613) 761-5256; Fax: (613) 761-5281; E-mail: rmcpherson@ottawaheart.ca.
1. Yusuf, S., Reddy, S., Ounpuu, S., and Anand, S. 2001. Global burden of cardiovascular diseases. I. General considerations, the epidemiologic transition, risk factors, and impact of urbanization. Circulation. 104:2746-2753.

2. Schildkraut, J.M., Myers, R.H., Cupples, L.A., Kiely, D.K., and Kannel, W.B. 1989. Coronary risk associated with age and sex of parental heart disease in the Framingham Study. Am. J. Cardiol. 64:555-559.

3. Greenland, P., et al. 2003. Major risk factors as antecedents of fatal and nonfatal coronary heart disease events. JAMA. 290:891-897.

4. Lusis, A.J. 2000. Atherosclerosis. Nature. 407:233-241.

5. Nora, J.J., Lortscher, R.H., Spangler, R.D., Nora, A.H., and Kimberling, W.J. 1980. Genetic-epidemiologic study of early-onset ischemic heart disease. Circulation. 61:503-508.

6. Broeckel, U., et al. 2002. A comprehensive linkage analysis for myocardial infarction and its related risk factors. Nat. Genet. 30:210-214.

7. Harrap, S.B., et al. 2002. Genome-wide linkage analysis of the acute coronary syndrome suggests a locus on chromosome 2. Arterioscler. Thromb. Vasc. Biol. 22:874-878.

8. Francke, S., et al. 2001. A genome-wide scan for coronary heart disease suggests in Indo-Mauritians a susceptibility locus on chromosome 16p13 and replicates linkage with the metabolic syndrome on 3q27. Hum. Mol. Genet. 10:2751-2765.

9. Chen, Q., et al. 2003. Genetic variation in lectinlike oxidized low-density lipoprotein receptor 1 (LOX1) gene and the risk of coronary artery disease. Circulation. 107:3146-3151.

10. Wang, Q., et al. 2004. Premature myocardial infarction novel susceptibility locus on chromosome 1P34-36 identified by genomewide linkage analysis. Am.J. Hum. Genet. 74:262-271.

11. Topol, E.J., et al. 2001. Single nucleotide polymorphisms in multiple novel thrombospondin genes may be associated with familial premature myocardial infarction. Circulation. 104:2641-2644.

12. Pajukanta, P., et al. 2000. Two loci on chromosomes 2 and $\mathrm{X}$ for premature coronary heart disease identified in early- and late-settlement populations of Finland. Am. J. Hum. Genet. 67:1481-1493.

13. Hauser, E.R., et al. 2004. A genomewide scan for early-onset coronary artery disease in 438 families: the GENECARD Study. Am. J. Hum. Genet. 75:436-447.

14. Lusis, A.J. 2003. Genetic factors in cardiovascular disease. 10 questions. Trends Cardiovasc. Med. 13:309-316.

15. Scheuner, M.T. 2003. Genetic evaluation for coronary artery disease. Genet. Med. 5:269-285.

16. Wang, L., Fan, C., Topol, S.E., Topol, E.J., and Wang, Q. 2003. Mutation of MEF2A in an inherited disorder with features of coronary artery disease. Science. 302:1578-1581.

17. McKinsey, T.A., Zhang, C.L., and Olson, E.N. 2002. MEF2: a calcium-dependent regulator of cell division, differentiation and death. Trends Biochem. Sci. 27:40-47.

18. Black, B.L., and Olson, E.N. 1998. Transcriptional control of muscle development by myocyte 
enhancer factor-2 (MEF2) proteins. Annu. Rev. Cell Dev. Biol. 14:167-196.

19. Naya, F.J., et al. 2002. Mitochondrial deficiency and cardiac sudden death in mice lacking the MEF2A transcription factor. Nat. Med. 8:1303-1309.

20. Bhagavatula, M.R.K., et al. 2004. Transcription factor MEF2A mutations in patients with coronary artery disease. Hum. Mol. Genet. 13:3181-3188.

21. European Molecular Biology Laboratory. 2004. PolyPhen: prediction of functional effect of human nonsynonymous SNPs. http://www.bork. embl-heidelberg.de/PolyPhen/.
22. Sunyaev, S., et al. 2001. Prediction of deleterious human alleles. Hum. Mol. Genet. 10:591-597.

23. Ramensky, V., Bork, P., and Sunyaev, S. 2002. Human non-synonymous SNPs: server and survey. Nucleic Acids Res. 30:3894-3900.

24. Miller, S.A., Dykes, D.D., and Polesky, H.F. 1988. A simple salting out procedure for extracting DNA from human nucleated cells. Nucleic Acids Res. 16:1215.

25. ELXR: exon resequencing primer design tool. http://mutation.swmed.edu/ex-lax.

26. Lansang, C., et al. 2004. Post-Sequencing BET
Cleanup Protocol. http://www.jgi.doe.gov/ sequencing/protocols/AutomatedBETCleanupSOP.doc.

27. Khan, A., and Jett, J. 2004. Cycle sequencing using BigDye v3.1: performed on fosmid DNA template. http://www.jgi.doe.gov/sequencing/protocols/ BigDyev3.1FosmidCycleSequencingSOP.doc.

28. Green, P. 2004. Phred, Phrap, Consed sequence analysis tools. http://www.phrap.org/phredphrapconsed.html.

29. Nickerson Group. 2003. PolyPhred. http://droog. mbt.washington.edu/PolyPhred.html. 